\title{
ABORDAGEM HÍBRIDA NO TRATAMENTO DO TUMOR DE GLOMUS CAROTÍDEO: RELATO DE EXPERIÊNCIA
}

\section{Ricardo Turra PERRONE ${ }^{1 *}$, Jaffer Tardim CARDERELLI ${ }^{1}$, Leonardo Barros PICININNI, Alexandre Funes BASTOS ${ }^{1} \&$ Eugênio Carlos de Almeida TINOCO ${ }^{1,2}$}

1Hospital São José do Avaí. Itaperuna, Rio de Janeiro, Brasil. 2Universidade Iguaçu, campus V. Itaperuna, Rio de Janeiro, Brasil.

*Autor para correspondência: turraperrone@gmail.com

DOI: http://dx.doi.org/10.18571/acbm.150

\section{RESUMO}

O tumor glômico carotídeo é um tipo raro de tumor neuroendócrino que tem origem nas células da crista neural do sistema paraganglionar, sendo encontrados numa frequência de $0,012 \%$ de todos os tumores de cabeça e pescoço. O que o torna ponto de discussão entre cirurgiões vasculares e endovasculares sobre os melhores tipos de abordagens para tratamento cirúrgico desta patologia. O presente trabalho trata-se de um estudo observacional, do tipo transversal utilizando como metodologia de pesquisa um estudo quali-quantitativo com procedimentos metodológicos baseados em estudo de casos abordados no serviço de Cirurgia Vascular do Hospital São Jose do Avaí - Itaperuna - RJ, entre os anos de 2009 e 2017. Foram realizadas 6 abordagens hibridas de embolização percutânea associada a cirurgia aberta para tratamento desta afecção neste serviço. $\mathrm{O}$ resultado obtido mostra que a embolização pré-operatória, apesar de controversa, demonstrou ser um procedimento eficaz e seguro para o tratamento dos tumores de glômus carotídeo, com baixo índice de complicações neurológicas e hemorrágicas neste tipo de tratamento.

Palavras-chave: Carótida; Tumor Glômico; Embolização; Cirurgia.

\begin{abstract}
The carotid glomus tumor is a rare type of neuroendocrine tumor that originates in the cells of the neural crest of the paraganglionar system, being found in a frequency of $0.012 \%$ of all tumors of head and neck. This makes it a point of discussion between vascular and endovascular surgeons on the best types of approaches to surgical treatment of this pathology. The present study is a retrospective cross-sectional study using as a research methodology a qualitative and quantitative study with methodological procedures based on a case study in the vascular surgery department of the Hospital São José do Avaí - Itaperuna - RJ, between the years 2009 and 2017. Six hybrid approaches of percutaneous embolization associated with open surgery to treat this condition in this service were performed. The results show that preoperative embolization, despite being controversial, has been shown to be an effective and safe procedure for the treatment of carotid glomus tumors, with a low rate of neurological and hemorrhagic complications in this type of treatment.
\end{abstract}

Keywords: Carotid; Glomus Tumors; Embolization; Surgery.

\section{Introdução}

O tumor glômico carotídeo é um tipo raro de tumor neuroendócrino que tem origem nas células da crista neural do sistema paraganglionar. Histologicamente apresentam características semelhantes ao tecido do corpo carotídeo, localiza-se na maioria das vezes, na região pósteromedial da bifurcação carotídea, a ressecção cirúrgica pode ter consequências neurológicas e perda sanguínea importante devido à proximidade com estruturas nobres da região cervical (VEDOLIM, 2003; CACERES, 2014). Os paragangliomas foram denominados, primariamente, quimiodectomas. No entanto, o termo tumores do corpo carotídeo emergiu como o mais comum 
para designar os tumores localizados na bifurcação das artérias carótidas, sendo encontrados numa frequência de 0,012\% de todos os tumores de cabeça e pescoço, (ERICKSON, 2001; GIL, 2003; JUNIOR, 2016).

Entre os tumores do corpo carotídeo, apenas $2 \%$ são malignos e os benignos, excepcionalmente, se malignizam. Eles originam-se dos pequenos órgãos quimiorreceptores localizados na adventícia das bifurcações das artérias. O que o torna ponto de discussão entre cirurgiões vasculares e endovasculares sobre os melhores tipos de abordagens para tratamento cirúrgico desta patologia, com a necessidade ou não de embolização tumoral pré-operatória (MATTICARI, 1995; FRANÇA, 2003; GONZÁLEZ, 2006; JIMENEZ, 2006).

Caracteristicamente, os tumores do corpo carotídeo ocorrem frequentemente entre a terceira e sexta década de vida, mais comum no sexo feminino, e se apresenta como uma massa cervical, unilateral, indolor, de consistência fibroelástica, pulsátil, com mobilidade horizontal e fixação vertical devido à intima fixação ao corpo carotídeo (GULYA, 1993).

Histologicamente são similares ao feocromocitoma de adrenais, porém, apenas uma minoria é secretora de catecolaminas. A malignidade é observada em até $11 \%$ dos casos e o potencial metastático parece não estar relacionado ao tamanho do tumor. Tais tumores devem sempre ser diferenciadas de outras massas cervicais, como cisto braquial, tumores neurogênicos, aneurisma de carótida, metástases cervicais dentre outros. A biópsia de tumores do corpo carotídeo não deve ser realizada, pois pode provocar hemorragia de difícil controle. A confirmação diagnostica se dá por exames de imagem para adequada definição de estratégia de abordagem (BRINER et al., 1999).

Neste artigo, foram avaliados a eficácia e segurança da abordagem hibrida no tratamento do tumor de glômus carotídeo, sendo inicialmente realizada a embolização do tumor de corpo carotídeo e em um segundo tempo a ressecção e cirúrgica convencional.

\section{Relato de experiência}

Entre os anos de 2009 a 2017 o serviço de Cirurgia Vascular do Hospital São Jose do Avaí em Itaperuna, Rio de Janeiro realizou 6 abordagens hibridas para tratamento do tumor de glômus carotídeo. Nesse período o procedimento foi realizado em seis pacientes do sexo feminino com idade variando de 26 a 66 anos, na época da abordagem cirúrgica. Nenhum delas possuía histórico familiar de tumores paraganglionares e todas possuíam lesões unilaterais. Três eram portadoras de Diabetes Mellitus tipo 2; cinco hipertensas; uma tabagista; uma com DPOC.

O diagnóstico foi baseado em imagens de tomografia computadorizada convencional cervical, arteriografia e angiotomografia de carótidas e vertebrais.

As pacientes foram submetidas à embolização com microesferas via endovascular préoperatória, e operados de 2 a 5 dias após o procedimento. A embolização pré-operatória visa diminuir a vascularização tumoral, diminuição tumoral, e consequente sangramento durante o ato cirúrgico. Este motivo foi fundamental para a escolha desta técnica que foi empregada em todos os casos relatados nesse trabalho.

O tratamento definitivo e curativo é a ressecção cirúrgica, que consiste em retirar totalmente o tumor preservando as estruturas nobres desta região. Em 5 casos a cirurgia foi realizada dois dias após a embolização, e para 1 caso cinco dias após a embolização, devido arritmia cardíaca vigente no segundo dia pós embolização.

Foram realizadas nestes pacientes a anestesia loco regional, que possibilita fazer avaliações constantes do estado de consciência e indiretamente a perfusão cerebral nestes pacientes durante $\mathrm{o}$ ato operatório.

O acesso cirúrgico se assemelha a endarterectomia carotídea. Uma incisão longitudinal diagonal, que segue ao longo da borda anterior do músculo esternocleitomastoide, seguindo da borda inferior do lobo da orelha até a borda superior clavicular. 
O procedimento deve, sempre que possível, manter condições ideais de fluxo sanguíneo cerebral durante a cirurgia e no pós-operatório. Deve expor toda a massa, e durante a dissecção manter a integridade da artéria carótida comum, interna e externa mantendo a vascularização cervical anatomicamente inalterada.

Em nenhum dos casos tratados houve complicações durante e após as embolizações, assim como durante a cirurgia convencional, não houve histórico de lesões vasculares, neurológicas, sangramento significativo, transfusões sanguíneas e/ou outras intercorrências.

\section{Considerações finais}

A embolização pré-operatória apesar de controversa, demonstrou ser um procedimento eficaz e seguro para o tratamento dos tumores de glômus carotídeo, com baixo índice de complicações neurológicas e hemorrágicas. A técnica empregada deve ser definida pelo cirurgião a depender de cada caso, a depender do perfil, anatomia e biotipo do paciente assim como as características e localização das lesões.

\section{Referências}

CÁCERES, H.; SILVA, S.; AMARILlA, R.; LACONICH, D.; SOSA, K. Tumor de glomus carotídeo, Cir. Parag. 38, 35, 2014.

BRINER, H. R.; LINDER, T. E.; PAW, B.; FISCH, U. Long-term results of surgery for temporal bone paragangliomas. Laryngoscope, New York, v. 109, p. 577-583, 1999.

ERICKSON D, KUDVA YC, EBERSOLD MJ, THOMPSON GB, GRANT CS, VANHEERDEN JA. Benign Paragangliomas: clinical presentation and treatment outcomes in 236 patients. J Clin Endocrinol Metab. 86(11):5210, 2001.

FRANÇA LHG, BREDT CG, VEDOLIN A, BACK LA, STAHLKE JR. HJ. Tratamento cirúrgico do tumor do corpo carotídeo: J Vasc Br. 2(3):171, 2003.

GIL F. L.H, GOMES B. C, VEDOLIN A, BACK L.A, STAHLKE H.J JR. Surgical treatment of the carotid body tumor: a 30-year experience. Jornal Vascular Brasileiro. 2: 171, 2003.

GONZÁLEZ-FUEYO MJ, BALLESTEROS-POMAR M, DOMÍNGUEZ-BAHAMONDE J, ZARCO-CASTILLO J, FERNÁNDEZ SAMOS R. Tumores del glomus carotídeo: estudio de 11 años. Angíologia. 58(2):91, 2006.

GULYA, A. J. The glomus tumors and its biology. Laryngoscope, New York, v. 60, p. 7, 1993.

JIMÉNEZ C, COTE G, ARNOLD A. Review: Should patients with apparently sporadic pheochromocytomas or paragangliomas be screened for hereditary syndromes? J Clin Endocrinol Metab. 91 (8): 2851, 2006.

JUNIOR, N.M., SILVA, R.S. Tumor de corpo carotídeo (paraganglioma): relato de dois casos submetidos a tratamento cirúrgico. J Vasc Bras. Abr.-Jun.; 15(2):158, 2016.

MATTICARI S, CREDI, G, PRATESI C, BERTINI D. Diagnosis and surgical treatment of the carotid body tumors. J Cardiovasc Surg. 36:233, 1995.

VEDOLIM AC, SCHMITT CMA, BREDT CFG, BARROS MB, FRANÇA LHG, STAHLKE JR HJ. Tumor do corpo carotídeo: análise de 14 casos. RBM Rev Bras Med. 60(5):267, 2003. 